\section{Prevalência e fatores associados \\ a sintomas depressivos em adultos do sul do Brasil: estudo transversal de base populacional}

\section{Prevalence of depressive symptons and associated factors among Southern Brazilian adults: cross- sectional population-based study}

\section{Resumo}

Com o objetivo de identificar a prevalência de sintomas depressivos e examinar fatores associados em uma população adulta do sul do Brasil, foi realizado um estudo transversal de base populacional, incluindo 972 indivíduos, de ambos os sexos, idade entre 20 e 69 anos, moradores na zona urbana da cidade de Pelotas, Rio Grande do Sul. A seleção amostral teve os setores censitários do município como unidades amostrais primárias e os domicílios como unidades secundárias. O questionário incluiu variáveis socioeconômicas, comportamentais e nutricionais. As prevalências dos sintomas depressivos tristeza, ansiedade, falta de energia, falta de disposição, pensar no passado e preferir ficar em casa, na população de Pelotas, foram, respectivamente, de $29,4 \%, 57,6 \%, 37,4 \%, 40,4 \%, 33,8 \%$ e $54,3 \%$. Concluiu-se que as prevalências de sintomas depressivos foram elevadas e os indivíduos do sexo feminino, idade avançada, fumantes e obesos mostraram-se associadas à maioria dos desfechos. A depressão é um importante problema de saúde publica e o conhecimento de como a sintomatologia depressiva se distribui na população e os fatores associados à sua presença podem ajudar no melhor entendimento da fenomenologia dos transtornos depressivos e a traçar estratégias de prevenção e intervenção.

Palavras chave: Sintomas depressivos. Saúde mental. Epidemiologia. Estudos transversais.

\section{Airton José Rombaldi' \\ Marcelo Cozzensa da Silva' \\ Fernando Kratz Gazalle"II \\ Mario Renato Azevedo' \\ Pedro Curi Hallal ${ }^{\prime \prime \prime}$ \\ ' Programa de pós-graduação em Educação Física da Universidade Federal de Pelotas, Brasil \\ "Programa de pós-graduação em Epidemiologia da Universidade Federal de Pelotas, Brasil \\ "I'Programa de Transtornos Bipolares e Laboratório de Psiquiatria Molecular do Hospital de Clínicas de Porto Alegre, Brasil}

Correspondência: Airton José Rombaldi. PPG em Educação Física. Universidade Federal de Pelotas. Rua Luis de Camões 625, Pelotas, RS - CEP 96055-630. E-mail: rombaldi@brturbo.com.br 


\section{Abstract}

To identify the prevalence of depressive symptoms and examine associated factors in a Southern Brazilian adult population, a cross-sectional population-based study was carried out, including 972 subjects, men and women, aged 20 to 69 years, living in the urban area of Pelotas, Rio Grande do Sul. The sampling strategy relied on the census tracts of the city as primary sampling units and households as the secondary units. The questionnaire included socio-demographic, behavioral and nutritional variables. The prevalence of sadness, anxiety, loss of energy, lack of will to do things, thinking about the past, and wishing to stay at home were $29.4 \%, 57.6 \%, 37.4 \%, 40.4 \%, 33.8 \%$, and $54.3 \%$, respectively. Female gender, older ages, smokers and obese individuals showed association with depressive symptoms. Population-based studies using longitudinal designs may help to clarify the relationship between biopsychosocial variables and depressive symptoms.

Keywords: Depressive symptoms. Mental health. Epidemiology. Cross-sectional studies.

\section{Introdução}

Os transtornos mentais afetam $25 \%$ da população em alguma fase da vida, representando quatro das dez principais causas de incapacidade em todo o mundo. As doenças mentais respondem por $12 \%$ da carga global de doenças, com um crescimento previsto para $15 \%$ em 2020 . Os distúrbios mais comuns são transtornos depressivos, transtornos de uso de substâncias químicas e esquizofrenia ${ }^{1}$. De acordo com Murray \& Lopes $^{2}$, a depressão deverá ser a segunda doença mais comum no ano de 2020 , sendo superada apenas pelas doenças cardíacas.

A literatura mostra que os transtornos depressivos, em estudos de base populacional, atingem prevalência de $10 \%$ e incidência de $2 \%$ na população. A prevalência é alta independentemente do local do estudo, do instrumento utilizado e dos períodos de tempo para os quais a prevalência se aplica. Os fatores associados aos transtornos depressivos comumente encontrados são sexo feminino, baixas renda e escolaridade, idade de início entre 20 e 40 anos, pessoas divorciadas/separadas, viúvas ou que moram sozinhas, falta de suporte social, residentes em zona urbana e estresse crônico. Pessoas com depressão apresentam cerca de cinco vezes mais alguma forma de incapacidade do que indivíduos assintomáticos e, aqueles deprimidos, consultam e se hospitalizam mais por todas as causas ${ }^{3}$.

No Brasil as doenças mentais estão entre as principais causas de aposentadorias por invalidez na Previdência Social ${ }^{4}$. Wünsch Filho ${ }^{5}$ relata que os problemas psicológicos figuram entre as cinco doenças que mais provocaram afastamento temporário do trabalho, e o trabalho é apontado como causa da maioria dessas morbidades. Harpham et al. ${ }^{6}$ concluíram, após estudo de revisão, a necessidade de maiores investigações sobre o tema saúde mental, especialmente através de estudos populacionais. Maragno et al. ${ }^{7}$ salientam que, no Brasil, é pequeno o número de investigações epidemiológicas de base populacional na área de saúde mental.

Nesse sentido, o presente estudo tem 
por objetivo verificar a prevalência e os fatores associados a sintomas depressivos em uma população adulta da zona urbana do município de Pelotas, RS, Brasil.

\section{Métodos}

Foi conduzido um estudo transversal de base populacional na cidade de Pelotas (localizada no extremo sul do Rio Grande do Sul, com cerca de 340.000 habitantes) na forma de consórcio de pesquisa. Foi realizada uma amostragem por conglomerados. O Instituto Brasileiro de Geografia e Estatística (IBGE) divide a cidade em 404 setores censitário urbanos, dos quais foram sorteados aleatoriamente 40 para serem incluídos no estudo. Em cada setor sorteado, foi escolhido aleatoriamente um ponto de partida para a seleção das residências a serem visitadas. A primeira casa foi incluída e as próximas foram selecionadas de forma sistemática, respeitando-se um pulo de sete domicílios, até atingir 15 moradias em cada setor. No total, foram selecionados 600 domicílios para participar do estudo. Nesses, todos os moradores com idade entre $20 \mathrm{e}$ 69 anos foram incluídos na amostra, exceto aqueles com incapacidade mental ou física severa que os impedisse de responder o questionário.

Com a amostra obtida $(n=972)$, o estudo foi capaz de estimar a prevalência de $10 \%$ (com erro de \pm 2 pontos percentuais) de sintomas depressivos. Para explorar as associações entre investigação dos sintomas de depressão e fatores associados, a amostra garantiu poder superior a $80 \%$, utilizando-se erro alfa de $5 \%$, risco relativo a ser detectado de 2,3 e acréscimo de $10 \%$ para perdas e recusas.

Características demográficas, socioeconômicas e de saúde foram avaliadas por meio de um questionário padronizado. A variável cor da pele foi observada pelos entrevistadores e o nível econômico determinado segundo classificação da Associação Brasileira de Empresas de Pesquisa $(\mathrm{ABEP})^{8}$. Utilizou-se um ponto de corte de 150 minutos por semana para classificar os sujeitos como ativos ou sedentários. O estado nutricional foi determinado pelo índice de massa corporal (IMC), calculado a partir do peso e altura referidos.

Como desfechos do estudo foram avaliados os sintomas depressivos tristeza, ansiedade, falta de energia para as atividades do dia-a-dia, falta de disposição, pensar muito no passado e preferir ficar em casa. $\mathrm{O}$ instrumento utilizado para a mensuração das variáveis dependentes foi adaptado de um questionário idealizado por Gazalle et al. ${ }^{9}$. O mesmo foi desenvolvido para a investigação de alguns sintomas depressivos comumente encontrados na prática clínica em idosos, mas são também sintomas bastante comuns em adultos jovens e que muitas vezes não são investigados por outros instrumentos de avaliação de sintomas depressivos, como pensar muito no passado e ficar muito em casa. A utilização desse questionário adaptado também foi preferida pelas seguintes razões:

- número de perguntas: procurou-se elaborar um instrumento capaz de rastrear depressão em uma população com o mínimo de perguntas possíveis, facilitando sua inclusão em pesquisas similares;

- uso em atenção primária: o mesmo poderia ser facilmente aplicado na prática médica em geral. Todas as perguntas utilizaram como período de referência o último mês.

Os entrevistadores selecionados foram de ambos os sexos, com idade mínima de 18 anos e pelo menos ensino médio completo, tendo participado de um treinamento teórico-prático de 20 horas. Os questionários foram revisados por supervisores que estavam em contato permanente com os entrevistadores durante o trabalho de campo. Os mesmos realizaram controle de qualidade, por meio de revisitas a $25 \%$ da amostra. Os dados obtidos a partir dos questionários foram duplamente digitados no programa EpiInfo e as análises conduzidas no programa estatístico Stata 9.0.

$\mathrm{Na}$ análise bruta, foi verificada a relação entre os desfechos e as variáveis idade, cor 
da pele, sexo, nível econômico, tabagismo e estado nutricional. Para isso foram utilizados os testes de qui-quadrado para heterogeneidade e tendência linear. A análise multivariável foi realizada por meio de regressão de Poisson, com variância robusta, respeitando um modelo hierárquico de relações entre as variáveis ${ }^{10}$. No primeiro nível foram analisadas as variáveis idade, sexo e cor da pele; no segundo nível, a variável nível econômico; e no nível mais proximal foram incluídas as variáveis tabagismo e estado nutricional. Permaneceram no modelo final as variáveis que apresentaram na análise multivariável valor $\mathrm{p}<0,05$.

O projeto foi aprovado pelo Comitê de Ética em Pesquisa da Escola Superior de Educação Física da Universidade Federal de Pelotas e os dados foram coletados após consentimento informado dos sujeitos.

\section{Resultados}

A partir de 514 domicílios que tinham moradores na faixa etária estabelecida, o número de indivíduos elegíveis foi de 1.062 . Destes, 972 responderam ao questionário, o que representa uma taxa de não-respondentes de $8,5 \%$. A variável independente com maior número de valores ignorados foi o IMC, com 87 indivíduos não sabendo informar seus valores de peso e/ou altura.

A prevalência dos sintomas depressivos tristeza, ansiedade, falta de energia, falta de disposição, pensar no passado e ficar em casa, na população de Pelotas, foi, respectivamente, de $29,4 \%, 57,6 \%, 37,4 \%, 40,4 \%$, $33,8 \%$ e $54,3 \%$.

A Tabela 1 descreve a amostra e apresenta a prevalência dos sintomas depressivos conforme as variáveis independentes. A maioria da amostra estudada foi do sexo feminino (57\%) e menos de $1 / 4$ de cor da pele não branca. A faixa etária entre 20 e 29 anos foi a mais prevalente $(26,1 \%)$. Grande parte da amostra estudada $(41,5 \%)$ pertencia ao nível econômico C, 49,4\% nunca fumaram e cerca de $15,0 \%$ dos indivíduos foram classificados como obesos, a partir do IMC.

Em relação aos sintomas depressivos, as mulheres relataram maiores prevalências de quase todos os sintomas quando comparadas aos homens, com exceção dos sintomas pensar no passado e ficar em casa, os quais foram semelhantes. Quanto à idade, observou-se uma redução na prevalência dos sintomas ansiedade e falta de disposição à medida que aumentava a idade e o inverso foi encontrado no sintoma pensar no passado (Tabela 1).

Relação inversa das prevalências dos sintomas tristeza, pensar no passado e ficar em casa foi observada à medida que diminuía o nível econômico. Indivíduos fumantes apresentaram maiores prevalências de todos os sintomas depressivos, quando comparados a quem nunca fumou e a exfumantes. Indivíduos obesos foram mais prevalentes ao sintoma depressivo falta de energia (Tabela 1 ).

As Tabelas 2 e 3 apresentam as análises bruta e ajustada dos sintomas depressivos com as variáveis independentes em estudo. Em relação ao sintoma depressivo tristeza, as variáveis sexo, idade, nível econômico e tabagismo mostraram-se associadas ao desfecho. Mulheres apresentaram risco 80\% mais elevado que os homens; a idade mostrou associação significativa em forma de $\mathrm{U}$, sendo os indivíduos entre 50-59 anos os de maior risco (30\%); à medida que diminuiu o nível econômico, aumentou o risco desse sintoma. Fumantes atuais apresentaram $60 \%$ mais risco que não fumantes para esse desfecho (Tabela 2).

O sintoma depressivo ansiedade apresentou associação com as variáveis de exposição sexo, idade e tabagismo. Mulheres e fumantes apresentaram riscos aumentados para o sintoma quando comparados aos grupos de referência; a idade apresentou associação em forma de U (Tabela 2).

O sintoma depressivo falta de energia esteve associado a indivíduos do sexo feminino $(\mathrm{RP}=1,7)$ e fumantes $(\mathrm{RP}=1,4)$. A variável estado nutricional apresentou tendência de aumento do risco à medida que aumentam as categorias de IMC (Tabela 2).

A Tabela 3 apresenta as variáveis de exposição sexo, idade, tabagismo e estado 
Tabela 1 - Descrição da amostra $(n=972)$ do estudo e prevalência dos sintomas depressivos segundo variáveis independentes.

Table 1 - Description of the study sample $(n=972)$ and prevalence of depressive symptoms according to independent variables.

\begin{tabular}{|c|c|c|c|c|c|c|c|}
\hline Variáveis & $\mathrm{N}$ & $\begin{array}{c}\text { Tristeza } \\
(\%)\end{array}$ & $\begin{array}{c}\text { Ansiedade } \\
\text { (\%) }\end{array}$ & $\begin{array}{c}\text { Falta de } \\
\text { energia (\%) }\end{array}$ & $\begin{array}{c}\text { Falta de } \\
\text { disposição (\%) }\end{array}$ & $\begin{array}{l}\text { Pensar no } \\
\text { passado (\%) }\end{array}$ & $\begin{array}{l}\text { Ficar em } \\
\text { casa (\%) }\end{array}$ \\
\hline \multicolumn{8}{|l|}{ Sexo } \\
\hline Homens & 418 & 20,3 & 51,7 & 26,8 & 32,3 & 33,3 & 54,4 \\
\hline \multirow[t]{2}{*}{ Mulheres } & 554 & 36,2 & 62,0 & 45,4 & 47,7 & 35,4 & 54,3 \\
\hline & & $\mathrm{p}<0,001^{*}$ & $p=0,001^{*}$ & $\mathrm{p}<0,001^{*}$ & $\mathrm{p}<0,001^{*}$ & $p=0,5$ & $p=1,0$ \\
\hline \multicolumn{8}{|l|}{ Idade (anos) } \\
\hline $20-29$ & 254 & 23,0 & 66,0 & 39,9 & 44,5 & 28,7 & 52,6 \\
\hline $30-39$ & 195 & 25,8 & 56,0 & 35,6 & 41,0 & 30,3 & 52,6 \\
\hline $40-49$ & 247 & 34,2 & 54,5 & 39,0 & 43,3 & 35,6 & 54,3 \\
\hline $50-59$ & 173 & 36,1 & 59,3 & 37,8 & 37,0 & 37,0 & 57,9 \\
\hline \multirow[t]{2}{*}{60 ou mais } & 103 & 29,0 & 44,0 & 30,0 & 34,0 & 49,5 & 56,0 \\
\hline & & $\mathrm{p}=0,008^{\#}$ & $\mathrm{p}=0,002^{\#}$ & $p=0,2$ & $\mathrm{p}=0,05^{\#}$ & $\mathrm{p}<0,001^{\#}$ & $p=0,3$ \\
\hline \multicolumn{8}{|l|}{ Cor da pele } \\
\hline Branca & 797 & 28,2 & 57,4 & 36,5 & 40,8 & 33,5 & 53,6 \\
\hline \multirow[t]{2}{*}{ Não branca } & 175 & 34,9 & 58,3 & 41,7 & 42,3 & 38,9 & 57,7 \\
\hline & & $p=0,08$ & $p=0,8$ & $p=0,2$ & $p=0,7$ & $\mathrm{p}=0,2$ & $p=0,3$ \\
\hline \multicolumn{8}{|c|}{ Nível econômico } \\
\hline Classes A/B & 382 & 21,5 & 56,0 & 34,6 & 36,9 & 27,2 & 46,7 \\
\hline Classe C & 395 & 32,3 & 61,0 & 39,8 & 43,5 & 33,9 & 59,3 \\
\hline \multirow[t]{2}{*}{ Classes D/E } & 175 & 40,6 & 54,9 & 38,9 & 41,7 & 47,4 & 60,0 \\
\hline & & $\mathrm{p}<0,001^{\#}$ & $p=0,9$ & $p=0,2$ & $p=0,2$ & $\mathrm{p}=0,001^{\#}$ & $\mathrm{p}<0,001^{\#}$ \\
\hline \multicolumn{8}{|l|}{ Tabagismo } \\
\hline Nunca fumou & 480 & 25,8 & 55,1 & 35,2 & 38,3 & 30,8 & 49,6 \\
\hline Fumante atual & 276 & 39,6 & 67,5 & 45,6 & 50,0 & 40,2 & 63,1 \\
\hline \multirow[t]{2}{*}{ Ex-fumante } & 216 & 24,3 & 50,5 & 31,8 & 35,7 & 35,2 & 53,5 \\
\hline & & $\mathrm{p}<0,001^{*}$ & $\mathrm{p}<0,001^{*}$ & $p=0,003^{*}$ & $\mathrm{p}=0,001^{*}$ & $p=0,03^{*}$ & $p=0,002^{*}$ \\
\hline \multicolumn{8}{|c|}{ Estado nutricional } \\
\hline Eutrófico & 431 & 26,1 & 38,2 & 37,3 & 39,4 & 33,4 & 51,1 \\
\hline Sobrepeso & 321 & 27,7 & 55,5 & 32,0 & 32,7 & 32,7 & 55,5 \\
\hline \multirow[t]{2}{*}{ Obesidade } & 133 & 32,8 & 62,6 & 48,9 & 36,8 & 36,8 & 58,0 \\
\hline & & $p=0,2$ & $p=0,7$ & $p=0,003^{*}$ & $p=0,2$ & $p=0,6$ & $p=0,1$ \\
\hline
\end{tabular}

* $p$ de heterogeneidade / heterogeneity $p$

\# $\mathrm{p}$ de tendência linear / linear trend $p$

nutricional associadas ao desfecho falta de disposição. Mulheres e fumantes apresentaram risco $50 \%$ mais elevado para esse sintoma. À medida que diminuíram as categorias de idade aumentou o risco para o desfecho, sendo a categoria de 20-29 anos aquela que apresentou maior risco $(\mathrm{RP}=1,7)$. $\mathrm{O}$ estado nutricional mostrou associação direta com o desfecho, com maior razão de prevalência $(1,3)$ entre os obesos.

O desfecho pensar muito no passado apresentou associação direta com a idade e 
Tabela 2 - Análise bruta e ajustada das variáveis associadas aos sintomas depressivos tristeza, ansiedade e falta de energia no modelo de análise final.

Table 2 - Crude and adjusted analysis of variables associated with the depressive symptoms of sadness, anxiety, and lack of energy in the final analysis.

\begin{tabular}{|c|c|c|c|c|}
\hline \multirow[b]{2}{*}{ Variáveis } & \multicolumn{4}{|c|}{ Sintoma depressivo tristeza } \\
\hline & $\begin{array}{c}\text { Análise bruta } \\
\text { RP }\left(\mathrm{IC}_{95 \%}\right)\end{array}$ & Valor $\mathrm{p}$ & $\begin{array}{c}\text { Análise ajustada } \\
\operatorname{RP}\left({ }^{(} C_{95 \%}\right)\end{array}$ & Valor $p$ \\
\hline Sexo & & $<0,001^{*}$ & & $<0,001^{*}$ \\
\hline Homens & 1,0 & & 1,0 & \\
\hline Mulheres & $1,8(1,4$ a 2,2$)$ & & $1,8(1,4$ a 2,2$)$ & \\
\hline Idade (anos) & & $0,006^{* *}$ & & $0,007^{* * *}$ \\
\hline $20-29$ & $0,8(0,5$ a 1,2$)$ & & $0,9(0,6$ a 1,2$)$ & \\
\hline $30-39$ & $0,9(0,6$ a 1,3$)$ & & $0,9(0,6$ a 1,3$)$ & \\
\hline $40-49$ & $1,2(0,8$ a 1,7$)$ & & $1,2(0,9$ a 1,7$)$ & \\
\hline $50-59$ & $1,2(0,9$ a 1,8$)$ & & $1,3(0,9$ a 1,8$)$ & \\
\hline 60 ou mais & 1,0 & & 1,0 & \\
\hline Nível econômico & & $<0,001^{* *}$ & & $<0,001^{* *}$ \\
\hline Classes A/B & 1,0 & & 1,0 & \\
\hline Classe C & $1,5(1,2$ a 1,9$)$ & & $1,5(1,2$ a 1,9$)$ & \\
\hline Classes D/E & $1,9(1,4$ a 2,5$)$ & & $1,9(2,4$ a 2,4$)$ & \\
\hline Tabagismo & & $<0,001^{*}$ & & $<0,001^{*}$ \\
\hline Nunca fumou & 1,0 & & 1,0 & \\
\hline Fumante atual & $1,5(1,2$ a 1,9$)$ & & $1,6(1,2$ a 2,0$)$ & \\
\hline \multirow{2}{*}{ Ex-fumante } & $0,9(0,7$ a 1,2$)$ & & $0,9(0,7$ a 1,2$)$ & \\
\hline & \multicolumn{4}{|c|}{ Sintoma depressivo ansiedade } \\
\hline Sexo & & $0,002^{*}$ & & $0,001^{*}$ \\
\hline Homens & 1,0 & & 1,0 & \\
\hline Mulheres & $1,2(1,1$ a 1,3$)$ & & $1,2(1,1$ a 1,3$)$ & \\
\hline Idade (anos) & & $0,002^{* *}$ & & $0,001^{* * *}$ \\
\hline $20-29$ & $1,5(1,2$ a 1,9$)$ & & $1,5(1,2$ a 1,9$)$ & \\
\hline $30-39$ & $1,3(1,0$ a 1,6$)$ & & $1,3(1,0$ a 1,6$)$ & \\
\hline $40-49$ & $1,2(1,0$ a 1,6$)$ & & $1,2(1,0$ a 1,6$)$ & \\
\hline $50-59$ & $1,3(1,0$ a 1,7$)$ & & $1,3(1,1$ a 1,7$)$ & \\
\hline 60 ou mais & 1,0 & & 1,0 & \\
\hline Tabagismo & & $<0,001^{*}$ & & $<0,001^{*}$ \\
\hline Nunca fumou & 1,0 & & 1,0 & \\
\hline Fumante atual & $1,2(1,1$ a 1,4$)$ & & $1,3(1,1$ a 1,4$)$ & \\
\hline Ex-fumante & $0,9(0,8$ a 1,1$)$ & & $1,0(0,8$ a 1,2$)$ & \\
\hline
\end{tabular}

\begin{tabular}{|c|c|c|c|c|}
\hline & Sintoma depres & falta de en & para as ativida & lo dia-a-dia \\
\hline Sexo & & $<0,001^{*}$ & & $<0,001^{*}$ \\
\hline Homens & 1,0 & & 1,0 & \\
\hline Mulheres & $1,7(1,4$ a 2,0$)$ & & $1,7(1,4$ a 2,0$)$ & \\
\hline Tabagismo & & $0,002^{*}$ & & $<0,001^{*}$ \\
\hline Nunca fumou & 1,0 & & 1,0 & \\
\hline Fumante atual & $1,3(1,1$ a 1,5$)$ & & $1,4(1,2$ a 1,7$)$ & \\
\hline Ex-fumante & $0,9(0,7$ a 1,1$)$ & & $0,9(0,7$ a 1,1$)$ & \\
\hline Estado nutricional & & $0,002^{*}$ & & $0,002^{* *}$ \\
\hline Eutrófico & 1,0 & & 1,0 & \\
\hline Sobrepeso & $0,9(0,7$ a 1,0$)$ & & $0,9(0,8$ a 1,2$)$ & \\
\hline Obesidade & $1,3(1,1$ a 1,6$)$ & & $1,4(1,1$ a 1,7$)$ & \\
\hline
\end{tabular}


Tabela 3 - Análise bruta e ajustada dos sintomas depressivos falta de disposição, pensar muito no passado e preferir ficar em casa com variáveis sociodemográficas, econômica, comportamental e nutricional.

Table 3 - Crude and adjusted analysis of the depressive symptoms of lack of will to do things, thinking a lot about the past, and preferring to stay home with sociodemographic, economic, behavioral, and nutritional variables.

\begin{tabular}{|c|c|c|c|c|}
\hline \multirow[b]{2}{*}{ Variáveis } & \multicolumn{4}{|c|}{ Sintoma depressivo falta de disposição } \\
\hline & $\begin{array}{c}\text { Análise bruta } \\
\operatorname{RP}\left(\mathrm{IC}_{95 \%}\right)\end{array}$ & Valor $p$ & $\begin{array}{c}\text { Análise ajustada } \\
\text { RP }\left(\mathrm{IC}_{95 \%}\right)\end{array}$ & Valor $\mathrm{p}$ \\
\hline Sexo & & $<0,001^{*}$ & & $<0,001^{*}$ \\
\hline Homens & 1,0 & & 1,0 & \\
\hline Mulheres & $1,5(1,3$ a 1,7$)$ & & $1,5(1,3$ a 1,8$)$ & \\
\hline Idade (anos) & & $0,05^{* *}$ & & $0,03^{* *}$ \\
\hline $20-29$ & $1,3(1,0$ a 1,8$)$ & & $1,7(1,0$ a 1,9$)$ & \\
\hline $30-39$ & $1,2(0,9$ a 1,7$)$ & & $1,2(0,9$ a 1,7$)$ & \\
\hline $40-49$ & $1,3(0,9$ a 1,7$)$ & & $1,3(1,0$ a 1,8$)$ & \\
\hline $50-59$ & $1,1(0,8$ a 1,5$)$ & & $1,1(0,8$ a 1,6$)$ & \\
\hline 60 ou mais & 1,0 & & 1,0 & \\
\hline Tabagismo & & $<0,001^{* *}$ & & $<0,001^{*}$ \\
\hline Nunca fumou & 1,0 & & 1,0 & \\
\hline Fumante atual & $1,3(1,1$ a 1,5$)$ & & $1,5(1,3$ a 1,8$)$ & \\
\hline Ex-fumante & $0,9(0,8$ a 1,1$)$ & & $1,1(0,9$ a 1,4$)$ & \\
\hline Estado nutricional & & 0,2 & & $0,01 * *$ \\
\hline Eutrófico & 1,0 & & 1,0 & \\
\hline Sobrepeso & $1,0(0,8$ a 1,2$)$ & & $1,1(0,9$ a 1,3$)$ & \\
\hline \multirow[t]{2}{*}{ Obesidade } & $1,2(1,0$ a 1,5$)$ & & $1,3(1,0$ a 1,7$)$ & \\
\hline & \multicolumn{4}{|c|}{ Sintoma depressivo pensar muito no passado } \\
\hline Idade (anos) & & $<0,001^{* *}$ & & $<0,001^{* *}$ \\
\hline $20-29$ & $0,6(0,4$ a 0,4$)$ & & $0,6(0,4$ a 0,8$)$ & \\
\hline $30-39$ & $0,6(0,4$ a 0,8$)$ & & $0,6(0,4$ a 0,4$)$ & \\
\hline $40-49$ & $0,7(0,6$ a 1,0$)$ & & $0,7(0,6$ a 1,0$)$ & \\
\hline $50-59$ & $0,8(0,6$ a 1,0$)$ & & $0,8(0,6$ a 1,0$)$ & \\
\hline 60 ou mais & 1,0 & & 1,0 & \\
\hline Nível econômico & & $<0,001$ & & $<0,001^{* *}$ \\
\hline Classes A/B & 1,0 & & 1,0 & \\
\hline Classe C & $1,2(1,0$ a 1,5$)$ & & $1,3(1,1$ a 1,6$)$ & \\
\hline \multirow[t]{2}{*}{ Classes D/E } & $1,7(1,4$ a 2,2$)$ & & $1,7(1,4$ a 2,1$)$ & \\
\hline & \multicolumn{4}{|c|}{ Sintoma depressivo preferir ficar em casa } \\
\hline Nível econômico & & $<0,001^{* *}$ & & $<0,001^{* *}$ \\
\hline Classes A/B & 1,0 & & 1,0 & \\
\hline Classe $\mathrm{C}$ & $1,3(1,1$ a 1,5$)$ & & $1,3(1,1$ a 1,4$)$ & \\
\hline Classes D/E & $1,3(1,1$ a 1,5$)$ & & $1,3(1,1$ a 1,5$)$ & \\
\hline Tabagismo & & $<0,001^{*}$ & & $<0,001^{*}$ \\
\hline Nunca fumou & 1,0 & & 1,0 & \\
\hline Fumante atual & $1,3(1,1$ a 1,4$)$ & & $1,3(1,2$ a 1,5$)$ & \\
\hline Ex-fumante & $1,1(0,9$ a 1,3$)$ & & $1,1(0,9$ a 1,3$)$ & \\
\hline Estado nutricional & & 0,1 & & $0,04^{* *}$ \\
\hline Eutrófico & 1,0 & & 1,0 & \\
\hline Sobrepeso & $1,1(0,9$ a 1,2$)$ & & $1,1(1,0$ a 1,3$)$ & \\
\hline Obesidade & $1,1(1,0$ a 1,4$)$ & & $1,2(1,0$ a 1,4$)$ & \\
\hline
\end{tabular}


inversa com o nível econômico. O sintoma depressivo preferir ficar em casa mostrouse associado inversamente com o nível econômico e diretamente com o estado nutricional. Fumantes atuais apresentaram maior risco (Tabela 3).

\section{Discussão}

O presente estudo encontrou uma elevada prevalência de sintomas depressivos em amostra representativa de adultos residentes da cidade de Pelotas. A análise multivariável indicou que, em geral, indivíduos do sexo feminino, com idades mais avançadas, de baixo nível econômico, fumantes atuais e inativos, estão em maior risco de apresentarem os sintomas em estudo.

A utilização do instrumento escolhido pode ter implicado em algumas limitações:

- falta de comparabilidade;

- carência de validação: o instrumento utilizado não foi validado e, portanto, existem dúvidas quanto à sua habilidade em detectar quadros depressivos;

- diagnóstico de depressão: assim como outros instrumentos utilizados em estudos populacionais, existem dúvidas sobre a habilidade do questionário em diferenciar entre sintomas depressivos ou situações de vida estressante.

O maior risco de sintomas depressivos no sexo feminino, já a partir da adolescência, reflete um dado bastante conhecido na epidemiologia da depressão ${ }^{3,11-15}$. Esse achado é especialmente relevante nesse estudo de base populacional, porque refuta a idéia de que a depressão é mais diagnosticada em mulheres porque elas procuram mais os serviços de saúde, uma vez que o presente estudo foi realizado em domicílios da comunidade. Em relação a esse tópico, Justo \& Kalil ${ }^{16}$ citam possíveis explicações para esse maior risco, tais como questões socioculturais relacionadas com experiências adversas e atributos psicológicos e fisiológicos associados com maior vulnerabilidade a eventos estressantes.

Observou-se que quanto maior a idade dos indivíduos, maior a prevalência do sintoma depressivo pensar muito no passado. Esse achado concorda com estudo de Costa et al. ${ }^{17}$, e Lima ${ }^{3}$, os quais verificaram que a freqüência de distúrbios psiquiátricos menores aumentou com a idade em função do envelhecimento estar associado a maior exposição a doenças crônicas não transmissíveis e a eventos psicossociais como, por exemplo, luto, separações conjugais, perda de emprego. Essa associação positiva entre depressão e idade parece diferir de estudos com os critérios mundiais de depressão, que mostram que os idosos têm uma prevalência de depressão mais baixa que os adultos com menos idade. Essa tendência de redução na prevalência nos mais velhos foi observada nos sintomas depressivos ansiedade e falta de disposição, o que concorda com a literatura ${ }^{18}$.

A associação da variável nível econômico com a prevalência dos sintomas depressivos tristeza, pensar no passado e preferir ficar em casa mostra claramente a relação inversa com a posição social dos indivíduos. Como, em relação à temporalidade, a variável nível econômico precede os sintomas depressivos na hierarquia de determinação, esses achados reforçam a hipótese de que as condições de vida sejam determinantes para o aparecimento desses distúrbios. Tais resultados condizem com padrões encontrados em estudos realizados no Brasil ${ }^{3,19,20}$ e no exterior ${ }^{15,21,22}$.

Ser fumante mostrou-se associado a todos os sintomas depressivos, com exceção de pensar muito no passado, resultado consistente ao observado em outros estudos $^{13,23}$. No entanto, o fator temporal, de difícil determinação em estudos transversais, não permite estabelecer a relação de causa-efeito.

Estudos epidemiológicos têm mostrado forte relação entre obesidade e sintomas depressivos $^{24}$. Em nosso estudo elevados índices de massa corporal estiveram associados aos sintomas depressivos falta de energia, falta de disposição e preferir ficar em casa. Apesar desse achado, revisão sistemática recente descreve fracas evidências 
que suportam a hipótese de que a obesidade aumenta a incidência de depressão, existindo poucos estudos de coorte prospectiva de alta qualidade. A associação entre obesidade e depressão tem sido assumida a partir de resultados de estudos transversais, os quais podem ser acometidos de causalidade reversa ${ }^{25}$.

Algumas limitações devem ser apontadas. O delineamento transversal pode implicar em causalidade reversa entre o desfecho e as variáveis de exposição tabagismo e estado nutricional. Além disso, o instrumento utilizado para determinar a presença dos sintomas depressivos, apesar de coletar informações independentes sobre cada uma das variáveis que compõem o estado depressivo, não é validado. No entanto, ao revisarmos a literatura, encontramos uma série de instrumentos utilizados, validados ou não, para determinar a presença de sintomas depressivos, os quais dificultam a comparabilidade entre os resultados. Os pontos fortes do estudo são baixo índice de perdas e recusas, a amostra representativa $\mathrm{e}$ o criterioso cuidado metodológico.

O presente estudo identificou que os indivíduos do sexo feminino, com idade elevada, fumantes e obesos foram os que apresentaram maior probabilidade de apresentar os sintomas depressivos. Nesse sentido, políticas públicas de prevenção para esse público são necessárias e a realização de estudos de base populacional utilizando delineamentos longitudinais pode ajudar a fortalecer a relação de temporalidade entre importantes variáveis, como estado nutricional e tabagismo, com sintomas depressivos. Além disso, a validação do instrumento empregado no presente estudo seria um importante esforço para se dispor de um questionário simples, possível de ser utilizado tanto em ambiente de pesquisa de campo quanto na prática em atenção primária.

\section{Referências}

1. WHO. World health report. Mental health: new understanding, new hope. World Health Organization; 2001.

2. Murray C, Lopez A. The Global Burden of Disease. Boston: Harvard School of Public Health; 1996.

3. Lima MS. Epidemiologia e impacto social. Rev Bras Psiquiatr 1999; 21.

4. Moura AAG, Carvalho EF, Silva NJC. Repercussão das doenças crônicas não-transmissíveis na concessão de benefícios pela previdência social. Cien Saude Colet 2007; 12: 1661-72.

5. Wünsch Filho V. Variações e tendências na morbimortalidade dos trabalhadores. In: Monteiro C (ed.). Velhos e novos males da saúde no Brasil. São Paulo: Hucitec; 2000. p. 289-330.

6. Harpham T, Reichenheim M, Oser R, Thomas E, Hamid $\mathrm{N}$, Jaswal S, et al. Measuring mental health in a costeffective manner. Health Policy Plan 2003; 18: 344-9.

7. Maragno L, Goldbaum M, Gianini RJ, Novaes HMD, César CLG. Prevalência de transtornos mentais comuns em populações atendidas pelo Programa Saúde da Família (QUALIS) no Município de São Paulo, Brasil. Cad Saúde Pública 2006; 22: 1639-48.
8. Associação Brasileira de Empresas de Pesquisa. Critério de classificação econômica Brasil. Disponível em http:// www.abep.org/novo/Content.aspx?ContentID=301. [Acessado em 6 de março de 2010]

9. Gazalle FK, Lima MS, Tavares BF, Hallal PC. Sintomas depressivos e fatores associados em população idosa no Sul do Brasil. Rev Saúde Pública 2004; 38: 365-71.

10. Victora C, Huttly S, Fuchs S, Olinto M. The role of conceptual frameworks in epidemiological analysis: a hierarchical approach. Int J Epidemiol 1997; 26: 224-7.

11. Piccinelli M, Wilkinson G. Gender differences in depresion: critical review. Br J Psychiatry 2000; 177: 48692.

12. Patela V, Araya R, Lima M, Ludermir A, Todd C. Women, poverty and common mental disorders in four restructuring societies. Soc Sci Med 1999; 49: 1461-71.

13. Lindeman S, Hämäläinen J, Isometsä E, Kaprio J, Poikolainen K, Heikkinen M, et al. The 12-month prevalence and risk factors for major depressive episode in Finland: representative sample of 5993 adults. Acta Psychiatr Scan 2000; 102: 178-84.

14. Kantomaa MT, Tammelin TH, Ebeling HE, Taanila AM. Emotional and Behavioral Problems in Relation to Physical Activity in Youth. Med Sci Sports Exerc 2008; 40: 1749-56. 
15. Araya R, Rojas G, Fritsch R, Acuña J, Lewis G. Common mental disorders in Santiago, Chile. Br J Psychiat 2001; 178: 228-33.

16. Justo LP, Calil HM. Depressão: o mesmo acometimento para homens e mulheres? Rev Psiq Clín 2006; 33: 74-9.

17. Costa JSD, Menezes AMB, Olinto MTA, Gigante DP, Macedo S, Britto MAP, et al. Prevalência de distúrbios psiquiátricos menores na cidade de Pelotas, RS. Rev Bras Epidemiol 2002; 164: 164-73.

18. Fiske A, LoebachWetherell J, Gatz M. Depression in older adults. Annu Rev Clin Psychol 2009; 5: 363-89.

19. Ludermir AB, Filho DAM. Condições de vida e estrutura ocupacional associadas a transtornos mentais comuns. Rev Saúde Pública 2002; 36: 213-21.

20. Ludermir AB. Inserção produtiva, gênero e saúde mental. Cad Saúde Pública 2000; 16: 647-59.

21. Weich S, Lewis G. Material standard of living, social class, and the prevalence of the common mental disorders in Great Britain. J Epidemiol Community Health 1998; 52: 8-14.
22. Strawbridge WJ, Deleger S, Roberts RE, Kaplan GA. Physical activity reduces the risk of subsequent depression for older adults. Am J Epidemiol 2002; 156: 328-34.

23. Diaz F, James D, Botts S, Maw L, Susce M, Leon Jd. Tobacco smoking behaviors in bipolar disorder: a comparison of the general population, schizophrenia, and major depression. Bipolar Disord 2009; 11: 154-65.

24. Rosmond R, Lapidus L, Marin P, Bjorntorp P. Mental distress, obesity and body fat distribution in middleaged men. Obes Res 1996; 4: 245-52.

25. Atlantis E, Baker M. Obesity effects on depression: systematic review of epidemiological studies. Int J Obes 2008; 32: 881-91.

Recebido em: 17/08/09

Versão final reapresentada em: 30/06/10

Aprovado em: 23/07/10 